\title{
Simplified assay for enrichment of primed human Th17 and Tc17 lymphocytes from peripheral blood
}

\author{
Pradeep K. Dagur ${ }^{1 *}$, Elena Stansky ${ }^{1}$, Ankit Saxena ${ }^{1}$, Angélique Biancotto ${ }^{2}$ and John Philip McCoy Jr ${ }^{1 *}$
}

\begin{abstract}
Background: Interleukin-17A (IL-17A) is a potent pro-inflammatory cytokine that has been implicated in the pathogenesis of various autoimmune diseases. The production of IL-17A is commonly associated with subsets of CD4+ T cells (Th17) and CD8+ T cells (Tc17). Identifying these subsets based on intracellular expression of IL-17 or transcription factor RORC precludes isolation of viable Th17 and Tc17 cells and there by limits studies involving cell-cell interaction or cellular functions. Therefore, identifying surface markers that can help in identifying and enriching these cells is important.
\end{abstract}

Results: We used MCAM as a surrogate marker to identify in vivo committed human Th17 and Tc17 subsets. By employing high-speed fluorescence activated cell sorting, we enriched IL-17A-producing subsets from human specimens without the need for in vitro polarization using exogenous cytokines. These subsets can be investigated, following sorting, using a variety of methods such as ELISA, ex vivo functional assays and next generation sequencing to gain insights into the role of human Th17 and Tc17 in health and disease.

Conclusion: We here demonstrate that both CD4+ T cells (Th17) and CD8+ T cells (Tc17) cell populations can be identified based on the surface expression of melanoma cell adhesion molecule (MCAM or CD146).

Keywords: MCAM, CD146, Th17, Tc17, Flow cytometry

\section{Background}

Several protocols have been developed to study the Th17 and Tc17 populations in vitro utilizing purified memory $\mathrm{T}$ cells stimulated with anti-CD3 and anti-CD28 antibodies in the presence of polarizing cytokines such as IL-23, IL-6, IL-1 $\beta$, and TGF- $\beta$ [1]. However, in vitro priming conditions may not correctly mimic the in vivo microenvironment for Th17 and Tc17 cell development, and thus the relevance to human immunity and physiology remains elusive. Therefore, there is an need for a simple, robust and reproducible protocol to purify and enrich in vivo primed human Th17 and Tc17 subsets, which can be applied to a variety of clinical specimens.

Melanoma cell adhesion molecule (MCAM, Muc18, or CD146) is an adhesion marker primarily expressed on

\footnotetext{
*Correspondence: dagurpk@nhlbi.nih.gov; mccoyj@nhlbi.nih.gov

${ }^{1}$ Flow Cytometry Core Facility, National Heart Lung and Blood Institute, National Institutes of Health, 10 Center Dr., Bldg. 10, Rm 8C104, Bethesda, Maryland 20892, USA

Full list of author information is available at the end of the article
}

human endothelial cells [2]. CD146 expression can be found on $2-3 \%$ of $\mathrm{T}$ cells in the peripheral blood of healthy human donors [3]. CD146 is co-expressed along with multiple surface antigens of the Th17 and Tc17 lineage such as CD161, CCR6, IL-23R, CCR5, CD26 and CD58 $[4,5]$. We found that CD146+ $\mathrm{T}$ cells have effector memory phenotype and readily adhere to endothelial monolayers [6, 7]. Moreover, CD146+ T cells display the transcriptomic and phenotypic signature of human Th17 and Tc17 subsets, and are elevated in various autoimmune and inflammatory diseases (notably IL-17A-mediated diseases) such as rheumatoid arthritis [8, 9], Crohn's disease [5], sarcoidosis [4, 5], Behcet's disease $[4,5]$, psoriasis $[10,11]$ and multiple sclerosis [12]. Importantly, CD146+ T cells do not need to be polarized with exogenous cytokines to display Th17 or Tc17 phenotype; they display the Th17 and Tc17 phenotype upon simple activation by CD3 and CD28 crosslinking $[4,5]$. This information allowed us to use CD146 as a

(C) The Author(s). 2019 Open Access This article is distributed under the terms of the Creative Commons Attribution 4.0 International License (http://creativecommons.org/licenses/by/4.0/), which permits unrestricted use, distribution, and 
biomarker to identify populations of in vivo primed Th17 and Tc17 cells.

\section{Results \\ Cell sorting strategy for Th17 and Tc17 cells}

Fluorescence activated cell sorting (FACS) allowed us to identify the Th17 and Tc17 populations using a combination of physical properties and surface staining expression (Fig. 1a and 1b). A portion of sorted cells were stained with anti human CD3 antibody to verify T cell nature and were subjected to Diff-Quick stain to confirm their lymphocytic cytology (Fig. 1c).

The sorted cells were briefly stimulated for $4 \mathrm{~h}$ in presence of brefeldin A and evaluated for IL-17 A and IFN- $\gamma$ secretion. Among all sorted populations, Th17 positive subset was found to have maximum frequency of IL-17A secreting cells without any polarization (Fig. 1d).

\section{Expression of Th17 and Tc17 signature genes and cytokines}

To confirm the observations made by immunophenotyping, real time PCR (QRTPCR) was performed on RNA isolated from freshly sorted un-stimulated cells. Sorted Th17 and Tc17 positive subsets showed increased expression of Th17-Tc17 signature transcription factor (ROR-C) and cytokines such as IL-17A (Fig. 2 a and b) compared to CD146- cell controls. In all PCR experiments, expression of CD146 mRNA was analyzed as a positive control to ensure that cells had been properly sorted. These data support that CD146 expression identifies Th17 and Tc17 signature transcripts.

We further stimulated the sorted CD146+ and CD146cells to evaluate the secretion of cytokines by sorted Th17 and Tc17+ control cells from peripheral blood of healthy donors. Sorted cells were stimulated for 5 days in vitro with plate bound anti-human CD3/CD28 without the addition of any exogenous polarizing cytokines. PMA, ionomycin and brefeldin-A were added for the final $4 \mathrm{~h}$ of incubation. The cells were stained and analyzed by flow cytometry to examine intracellular cytokine expression. These experiments showed that the CD146+ sorted cells expressed significantly more IL17A than the CD146-negative T cells (Fig. 3). These cells demonstrated expression of IFN- $\gamma$ in both the CD146 positive and negative fractions.

\section{Multiplex bead array (Luminex) analysis of cytokines secreted in vitro}

Culture supernatants were saved from the in vitro experiments in which sorted cells were stimulated with plate bound anti-human CD3/CD28 for 5 days, in order to assess the levels of cytokines secreted by Th17 and Tc17 positive and negative cells. Multiplex bead arrays for the analysis of cytokines in the supernatants from stimulated
Th17 and Tc17 cells revealed increased secreted IL-17A compared to the culture supernatants from the CD146$\mathrm{T}$ cells (Fig. $4 \mathrm{a}$ and $\mathrm{b}$ ), consistent with both the immunophenotyping and QRTPCR data.

\section{Discussion}

The human $\mathrm{T}$ cell lineage is highly heterogeneous, and various subsets of this lineage can be identified and purified using multi-parameter flow cytometric cell sorting. Conventionally, $\mathrm{T}$ cells are divided into helper and suppressor $\mathrm{T}$ cells and are primarily identified with surface expression of CD4 and CD8. Specifically, the Th17 and Tc17 subsets have garnered substantial interest in the past decade, and are regarded as playing a major role in cell recruitment, tissue inflammation, cytokine/chemokine production, and immunity against fungi and extracellular pathogens [13]. Human Th17 and Tc17 cells have been extensively documented as mediators of inflammation and linked to the pathogenesis of numerous autoimmune diseases such as inflammatory bowel disease, psoriasis and sarcoidosis $[14,15]$. Here we present a simple method to enrich Th17 / Tc17 cells for functional studies.

Th17 and Tc17 cells are typically identified by: (i) expression of the transcription factor retinoic acid receptor-related orphan receptor $\mathrm{C} 2$ (RORC2) (or the mouse ortholog RORyt), and (ii) by secretion of signature pleiotropic cytokines of the IL-17A [16-21]. Additionally, several surface antigens have been proposed to be surrogate markers of the Th17 subsets such as CD161, CCR6, IL-23R, CCR5, and CD26 [22-26]. However, there is no consensus regarding a single antigen or unique combination of antigens to phenotypically identify the Th17 and TC17 subsets, and cells purified on the basis of the markers listed above require in vitro polarization with exogenous cytokines before acquiring Th17 functional activities. This has been a limiting factor in the efforts to purify in vivo circulating human Th17/Tc17 cells, and there is currently no standardized method available to purify these subsets for enrichment and functional studies.

Our methodology has been tested and optimized for the enrichment and purification of both Th17 and Tc17 cells using whole blood, fresh and frozen PBMCs, and skin biopsies of human specimens. The flow cytometry staining panel includes the following: (i) CD45 to identify leukocytes and to exclude circulatory endothelial cells and non leukocyte cells, (ii) a dump channel containing CD19, CD33 and CD14 to exclude non-T cells (B-cells, monocytes/macrophages and neutrophils) that may express CD146 in the CD45+ population, (iii) CD4 and CD8 to identify $\mathrm{T}$ cell subsets, (iv) CD45RO to define subsets of memory cells in the CD4+ lineage, and (v) CD146 to identify Th17 and Tc17 cells. This panel intentionally avoids 

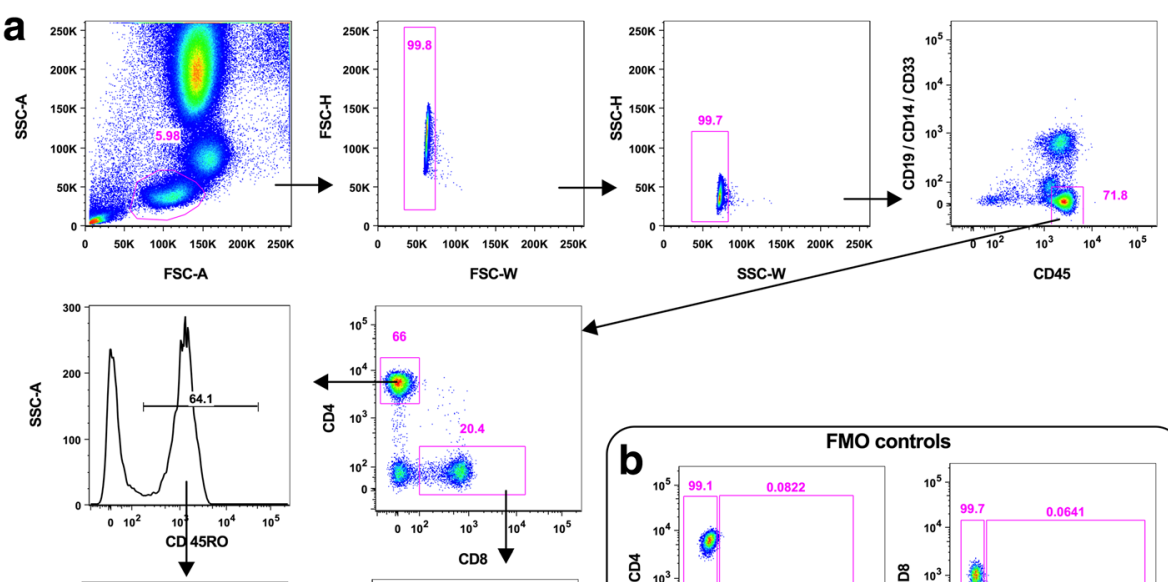

CD45
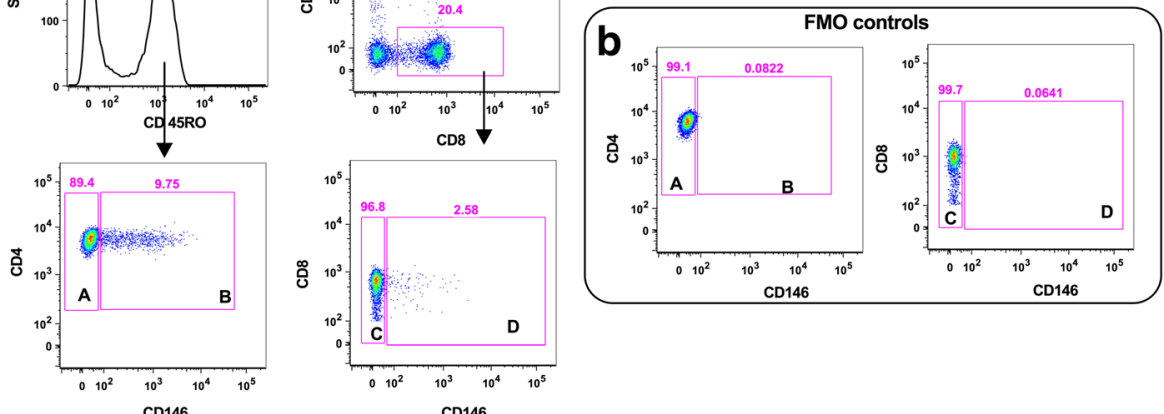

C
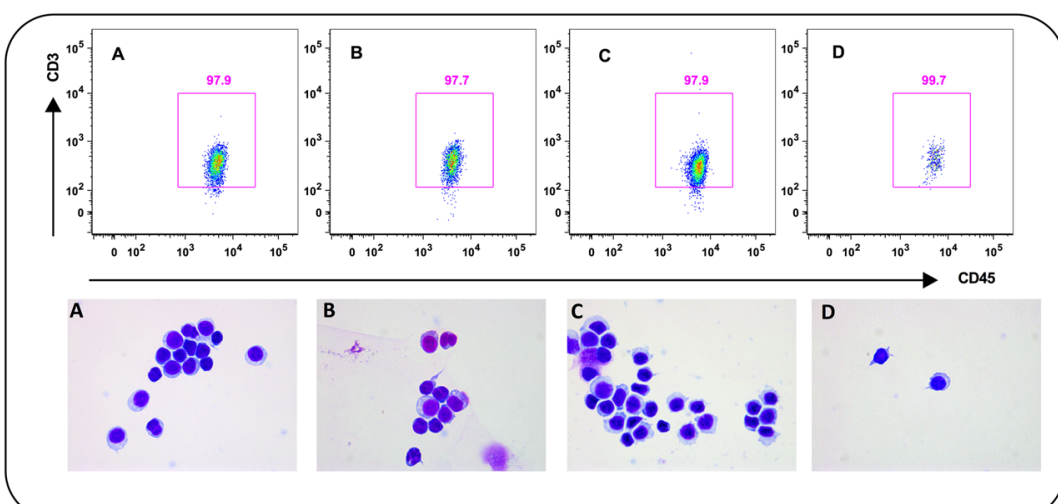

d
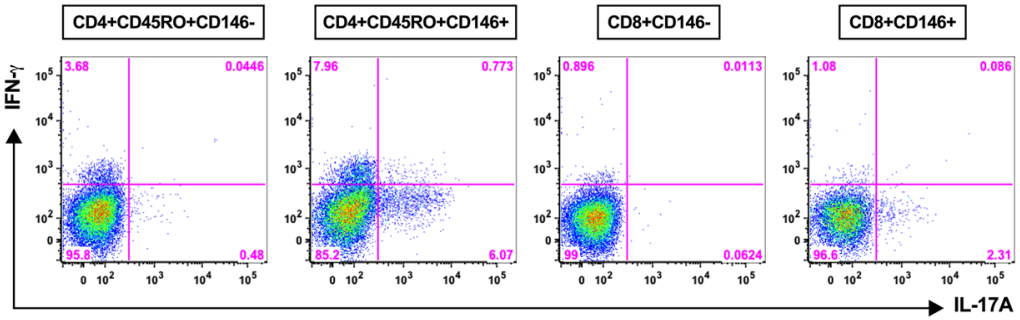

Fig. 1 Schematic representation of FACS plots showing strategy for sorting CD146+ Th17 and Tc17 cells. a Cells obtained after whole blood lysis were stained and sorted as CD45 + CD19-CD33-CD14-CD4 + CD45RO + CD146- (Th17 negative subset), CD45 + CD19-CD33-CD14-CD4 + CD45RO + CD146+ (Th17 positive subset), CD45 + CD19-CD33-CD14-CD8 + CD146- (Tc17 negative subset), and CD45 + CD19-CD33-CD14-CD8 + CD146+ (Tc17 positive subset). Lymphocytes were gated on forward and side scatter and doublets were removed. On gated CD45+ cells CD45RO + CD4 + CD146+ were gated for Th17 cells and CD8 + CD146+ as Tc17 cells. b Gating for CD146 was done on the basis of FMO controls. c Sorted cells were acquired to check purity and expression of CD3 antigen on sorted of populations. The cells were collected and stained with Diff-Quick cell staining kit to show lymphocyte morphology. d IL-17A and IFN-g secretion by freshly sorted Th17 and Tc17 positive and negative subset. As observed from the stimulated cells CD146+ CD4+ and CD146+ CD8+ cells were secreting IL-17A and IFN-g

the use of CD3 and CD28 antibodies to accommodate potential downstream experiments that may require $\mathrm{CD} 3$ and CD28 antigens for stimulation. However, we confirmed the presence of surface CD3 antigen on the sorted
Th17 cells, and control subsets via flow cytometry (Fig. 1c). Purified sorted subsets CD45 + CD19-CD33-CD14$\mathrm{CD} 4+\mathrm{CD} 45 \mathrm{RO}+\mathrm{CD} 146+$ (Th17) and CD45 + CD19CD33-CD14-CD8 + CD146+ (Tc17) be used downstream 

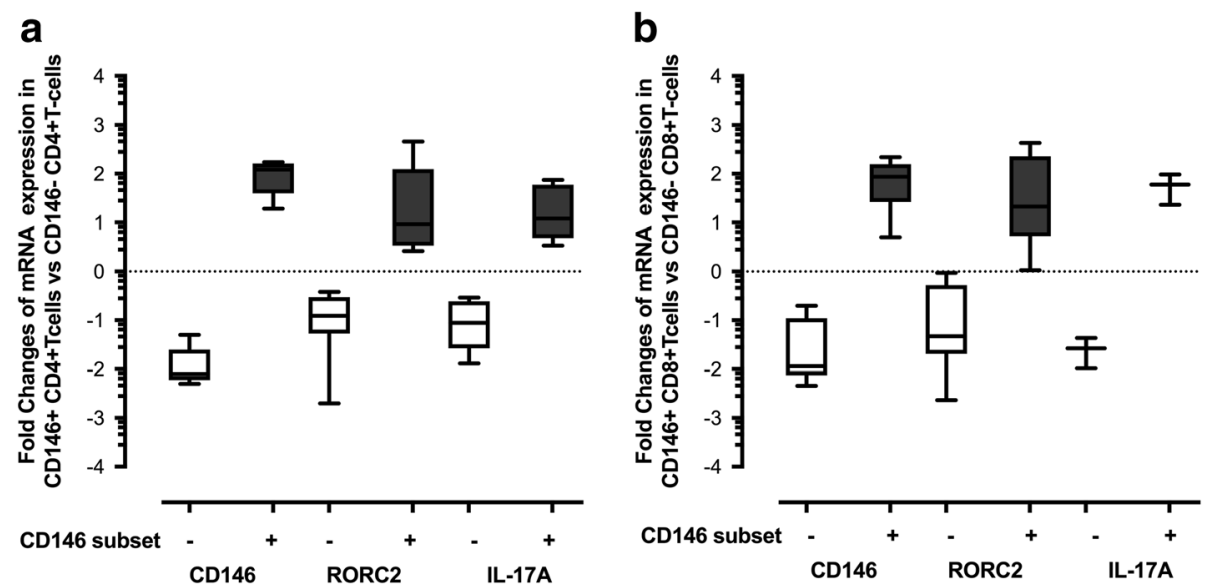

Fig. 2 QRTPCR of mRNA isolated from sorted CD4 + CD146+ and CD8 + CD146+ T cells: RNA was isolated from freshly sorted lymphocytes derived from healthy individuals $(n=5)$, and CDNA was prepared as described and QPCRs for CD146, RORc2 and IL-17A were performed. Fold changes were normalized mRNA levels with housekeeping gene ( $\beta$-actin) for (a) CD4 + CD45RO + CD146+ and (b) CD8 + CD146+ T cells were studied.compared to CD4+CD146- T cells and CD8 + CD146-T cells were studied

assays (such as transcriptome or proteomic analysis etc.) on Th17 and Tc17 subsets respectively, and CD146- subsets could serve as controls.

CD146 expression on human Tc17 subsets has been proven by us $[4,5,8,11]$, as well as others $[10,12]$. Our previous work in peripheral blood demonstrated that circulating CD146+ $\mathrm{T}$ cells display an effector memory phenotype, adhere efficiently to endothelium monolayer, and have a pro-inflammatory gene signature [6]. Furthermore, CD146+ T cells are primarily concentrated at the sites of inflammation, and display multiple Th17 and Tc17 signatures as found in the inflamed synovium of musculoskeletal disorders [8] and skin lesions [11]. Additionally, CD146 expression on CD4+ and CD8+ T cells can help in distinguishing Th17 and Tc17 mediated disease(s) [4].

This method allows for the enrichment of in vivo, primed human Th17 and Tc17 subsets, as well as their control subsets, from a variety of clinical specimens in a rapid, efficient, and highly reproducible manner. As this method allows for the purification of Th17 and Tc17 subsets primed in vivo, it avoids potential artifacts in downstream assays that could occur while skewing $\mathrm{T}$ cells in vitro towards
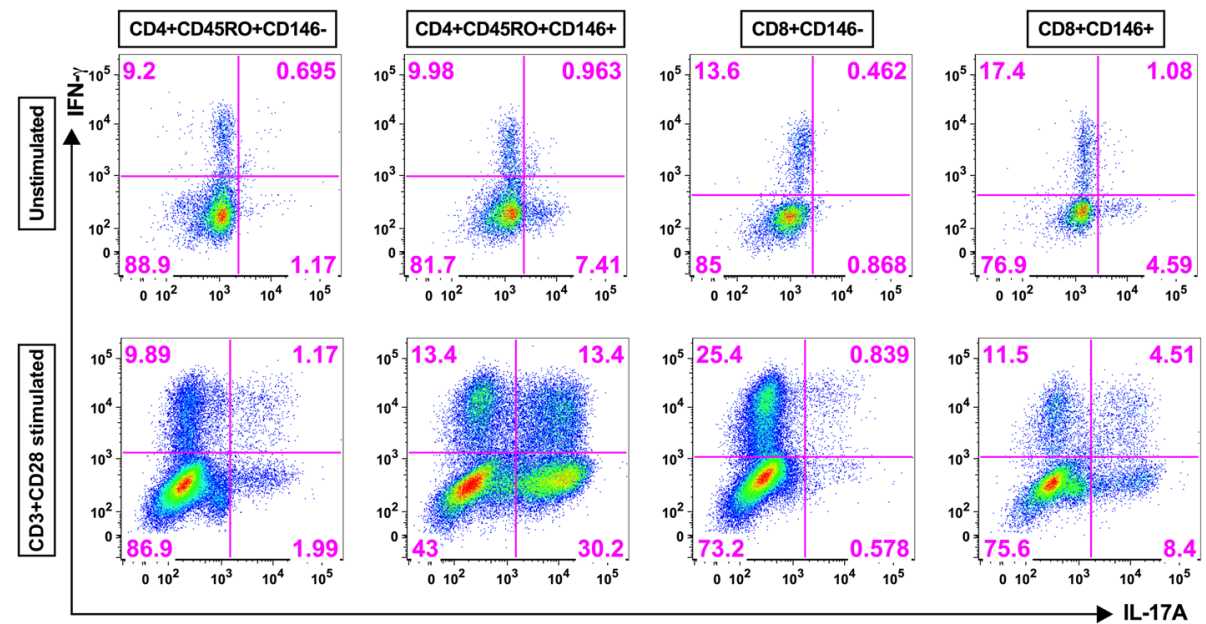

Fig. 3 IL-17A secreting cells are enriched within CD146+ CD4 and CD146+ CD8 subsets. Lymphocytes from whole blood lysis of healthy human peripheral blood were stained with mouse anti Human mAb CD4, CD8, CD45 RO and CD146 as described and sorted using flow cytometer. Sorted cells were finally washed with IMDM media and plated in 96 well flat bottom culture plate coated with purified anti-human CD3 and CD28 antibodies. Cells were incubated for 5 days; cultured cells were re-supplemented for the last $4 \mathrm{~h}$ of incubation with IMDM $+10 \%$ FCS and PMA/lonomycin in the presence of brefeldin-A and collected to perform intracellular cytokine staining for IL-17 and IFN- $\gamma$ cytokines 


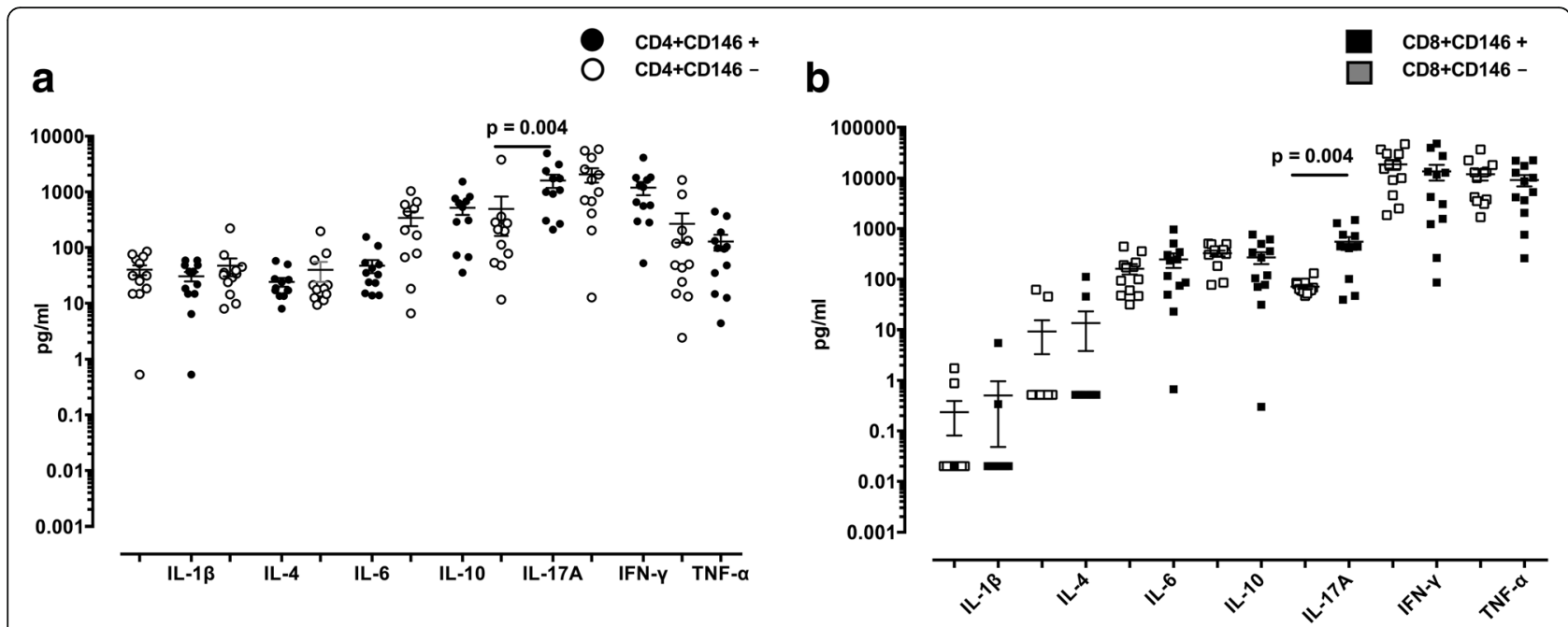

Fig. 4 CD146+CD4+ and CD8+CD146+ cells secrete significant amount of IL-17A. Lymphocytes from whole blood lysis of healthy human peripheral blood were stained with mouse anti Human mAb CD4, CD8, CD45 RO and CD146 and sorted. Sorted cells were finally washed with IMDM media and plated in 96 well flat bottom culture plate coated with purified antihuman CD3 and CD28 antibodies. Cells were incubated for 5 days and culture supernatant collected to perform multiplex using Luminex. Among analysis performed for various cytokines. Only IL-17 A was found to be significantly high among (a) CD146+CD4+ and (b) CD146+CD8+ cells. Samples were analyzes using Mann Whitney test and $p<0.05$ was considered significant

Th17 and Tc17 phenotype. The purified subsets may be used for downstream assays such as transcriptomic studies, genomic studies, functional studies, secretome studies, and so forth; which could be of great importance for basic human Th17/Tc17 biology. Further, this protocol not only allows for the enrichment and purification of Th17/Tc17subsets, but also has clinical relevance, as it can help in distinguishing whether a disease is Th17 mediated or Tc17 mediated in some diseases [4, 27].

Fluorescence activated cell sorting has various advantages, over other methods such as magnetic sorting, as it allows for the sorting of a population within a given combination of inclusion and exclusion cell surface markers, while simultaneously considering the physical properties of the cells. This protocol allows for the purification of Th17/Tc17 subsets along with matched memory non-Th17 population as controls.

Due to the differential expression of CD146 antigen between humans and mouse lymphocytes, this protocol can only be used for isolating human Th17/Tc17 subsets. In mice, CD146 staining is primarily present on natural killer (NK) cells, and T cells have not been shown to express the CD146 antigen [28]. This phenomenon may be due to the vast differences among human and mouse Th17 and Tc17 phenotypes $[29,30]$, or potential epitope mismatch and reagent unavailability to identify the same antigen on mouse subsets. Therefore, we recommend that this panel and protocol should only be used for sorting of Th17/ Tc17 subsets from human specimens.

\section{Conclusion}

We here present a novel method to identify and enrich human Th17 and Tc17 subsets, with least possible intervention or conventionally used conditional culture methods to isolate these cells. Identifying Th17 cells with the help of surface marker CD146 has an advantage over existing strategies as it provides more flexibility in isolating these cells from heterogenous lymphocyte population directly from whole blood and other sites of inflammation and thus helping to study these cells with minimal intervention.

\section{Materials and methods}

Blood samples and cell processing

To develop the protocol for isolation if Th17 and Tc17 cells using MCAM as a specific surface marker, 13 healthy donors (male $=8$, and females $=5$ ) age range:1845 yrs. were recruited. Peripheral blood samples were collected after approval by the institutional review board (IRB) and signing written informed consent by donors (protocol-07-H-0113).

\section{Sample preparation}

Twenty $\mathrm{ml}$ of fresh peripheral blood samples from healthy volunteers was collected in sodium-heparin vacutainers. Whole blood lysis was performed with ACK Lysis buffer (Blood: lysis buffer; 1:10, Quality Biological Inc). Cells were washed twice with sterile phosphate buffered saline $1 \mathrm{X}$ - without calcium chloride or magnesium chloride, $\mathrm{pH}$ 7.4, GIBCO-Invitrogen. All centrifugation steps were at $350 \mathrm{~g}$ for $5 \mathrm{~min}$ at $4{ }^{\circ} \mathrm{C}$. Cells thus 
obtained were stained with fluorochrome-conjugated mouse anti-human monoclonal antibodies: CD4 -FITC, CD8-APC-Cy7, CD14-APC, CD19-APC, CD33- APC, CD45- V450, CD45RO- PeCy5, CD146-PE (all from $\mathrm{BD}$ Biosciences). Cell viability was assessed using aqua fluorescent reactive dye (Life Technologies).

\section{Multiparametric FACS sorting}

Lymphocytes expressing CD45 antigen were identified, and CD45 + CD19-CD14-CD33- cells were selected to identify lymphocytes expressing CD4 and CD8 antigens. We omitted CD3 staining as sorted cells were to be stimulated later using anti-human CD3 and anti-human CD28 antibodies. CD4 bright cells are further gated on using CD45RO to select the CD4 T cell memory subset. The CD45RO gate was bypassed in the CD8+ T cell population, as there have been varying reports regarding the use of $\mathrm{CD} 45 \mathrm{RO}$ or CD45RA for selecting memory CD8+ T cells [31] (Figure 1.1). Positive staining for CD146 antigen was established using FMO controls, and CD4 + CD45RO+ cells and CD8+ cells are sorted depending on their positive or negative expression of CD146 (Figure: 1.2). Using this strategy, the sorted four subsets were as follows: CD45 + CD19-CD33CD14-CD4 + CD45RO + CD146- (Th17 negative subset), CD45 + CD19-CD33-CD14-CD4 + CD45RO + CD146+

(Th17 positive subset), CD45 + CD19-CD33-CD14-CD8 + CD146- (Tc17 negative subset), and CD45 + CD19-CD33CD14-CD8 + CD146+ (Tc17 subset). FACSAria SORP ${ }^{\text {тм }}$ sorter equipped with 355, 407, 488, 532 and 633 laser lines using DIVA ${ }^{\mathrm{mm}}$ 6.1.2 software (BD). FlowJo ${ }^{\mathrm{Tm}}$ software version 9.7.5 (Tree Star Inc.)

\section{In vitro stimulation}

Equal number of sorted CD8 + CD146 + and CD8 + CD146 - cells $(50,000$ to 100,000$)$ were cultured in $100 \mu \mathrm{l}$ of culture medium in 96 well $\mathrm{U}$ bottom culture plates with plate bound anti-human CD3 $(1 \mu \mathrm{g} / \mathrm{ml}$ clone OKT-3) and anti-human CD28 $(2.5 \mu \mathrm{g} / \mathrm{ml}$ clone CD28.2) in Iscove's Modified Dulbecco's Media (IMDM) supplemented with $10 \% \mathrm{FCS}$ and $1 \times$ antimycotic and antibiotic solution. Where possible, a control consisting of an equal number of cells from each sorted subset was kept in identical conditions, but without stimulation. Cytokine production was studied after CD8 + CD146 + and CD8 + CD146 - T cells were stimulated for 5 days as described. To use same cells to assess cytokines both in the culture supernatants as well as intracellularly, the culture supernatant was collected (5 days of culture) for Luminex analysis and then cultured cells were resupplemented for the last $4 \mathrm{~h}$ of incubation with IMDM $+10 \%$ FCS and PMA/Ionomycin in the presence of brefeldin-A (Leukocyte Activation Cocktail (BD)). Stimulated cells were then stained for both surface and intracellular antigens and analyzed and the culture supernatants kept at $-80^{\circ}$ until analysis of soluble cytokines by multiplex bead array.

\section{Gene expression}

RNA from freshly sorted unstimulated CD45 + CD19CD33-CD14-CD4 + CD45RO + CD146- (Th17 negative subset), CD45 + CD19-CD33-CD14-CD4 + CD45RO + CD146+ (Th17 positive subset) cells, CD45 + CD19CD33-CD14-CD8 + CD146- (Tc17 negative subset), and CD45 + CD19-CD33-CD14-CD8 + CD146+ (Tc17 positive subset). As described above, RNA was extracted using RNAqueous micro kit (Ambion, Austin, TX) according to the manufacturer's instructions. The Superscript cDNA synthesis kit (Invitrogen, Carlsbad, CA) was used to synthesize first strand cDNA as per the manufacturer's guidelines. cDNA was used as the template for amplification of the genes of interest and the control housekeeping genes ( $\beta$-Actin \# Hs99999903). Real time PCR (QRTPCR) was performed using a 7900-sequence detector (PE-Applied Biosystems, Norwalk, CT). Samples were analyzed in duplicate and, after normalizing the $\mathrm{Ct}$ values to housekeeping genes, fold changes in expression were calculated using the $\Delta \Delta \mathrm{Ct}$ (cycle threshold) method [22]. The primers obtained from Applied Biosystems were as follows: IL-17A \#Hs99999082_m1, ROR-C \#Hs01076112_ m1, and MCAM/CD146 \#Hs00174838_m1.

\section{Measurement of cytokines in culture supernatants}

Cytokines present in the supernatants of sorted CD45+ CD19-CD33-CD14-CD4 + CD45RO + CD146- (Th17 negative subset), CD45 + CD19-CD33-CD14-CD4 + CD45RO + CD146+ (Th17 positive subset), CD45 + CD19-CD33CD14-CD8 + CD146- (Tc17 negative subset), and CD45 + CD19-CD33-CD14-CD8 + CD146+ (Tc17 subset) were measured. Cells stimulated with plate bound anti-human CD3 and anti-human CD28 antibodies for 5 days was performed by multiplex bead arrays using kits for Luminex assays (HCYTOMAG-60 K-11 Human Cytokine Magnetic Millipore kit, Bellerica, MA) according to the manufacturer's instructions. Acquisition and analysis were performed on a Luminex-100 instrument using Bio-plex 6.1 software (Bio-Rad, Hercules, CA).

\section{Statistical analyses}

Data obtained with cells from one donor were considered as one experiment $(n)$. Statistical analyses were performed using the GraphPad Prism 6.0 software (La Jolla, CA), including the calculation of mean, standard errors of the mean (SEM), and $p$ values. Within one experiment, data were analyzed using a paired, two tailed, nonparametric Mann-Whitney (Wilcoxon rank sum) tests were used to analyze data. The significance level was set as $p \leq 0.05$, and the $p$ values were calculated for each series of experiments. 


\section{Acknowledgements}

Paula Schumm for recruitment of healthy volunteer Author's duly acknowledge Ann Wiliams and Liegh Samsel for their suggestions on operational aspects of cell sorter.

\section{Authors' contributions}

Design of study: PKD, JPM. Performed experiments: PKD, ES, AB, JPM. Writing manuscript PKD, AS, ES, AB, AW, JPM. Flow cytometry technical support: PKD, JPM. All authors read and approved the final manuscript.

\section{Funding}

Funding for this study was provided by the Division of Intramural Research National Heart, Lung, and Blood Institute, National Institutes of Health, Bethesda, MD.

\section{Availability of data and materials}

All data generated or analyzed during this study are included in this published article.

\section{Ethics approval and consent to participate}

This study was carried out in accordance with National Heart Lung and Blood Institute (NHLBI) institutional review board in accordance with the Declaration of Helsinki. All study participants provided written informed consent (protocol-07-H-0113).

\section{Consent for publication}

Not applicable.

\section{Competing interests}

Author Angélique Biancotto is employed by company Precision Immunology, Sanofi, MA, USA. The authors declare that they have no competing interests.

\section{Author details}

${ }^{1}$ Flow Cytometry Core Facility, National Heart Lung and Blood Institute, National Institutes of Health, 10 Center Dr., Bldg. 10, Rm 8C104, Bethesda, Maryland 20892, USA. ${ }^{2}$ Lab Head Immune Profiling, Precision Immunology, Immunology \& Inflammation Research Therapeutic Area, 270 Albany Street, Office\# 2212-A, Cambridge, MA 02139, USA.

\section{Received: 16 April 2019 Accepted: 30 May 2019}

\section{Published online: 02 July 2019}

\section{References}

1. Veldhoen M, Hirota K, Christensen J, O'Garra A, Stockinger B. Natural agonists for aryl hydrocarbon receptor in culture medium are essential for optimal differentiation of Th17 T cells. J Exp Med. 2009:206:43-9.

2. Bardin N, Anfosso F, Masse JM, Cramer E, Sabatier F, Le Bivic A, Sampol J, Dignat-George F. Identification of CD146 as a component of the endothelial junction involved in the control of cell-cell cohesion. Blood. 2001;98:3677-84.

3. Elshal MF, Khan SS, Takahashi Y, Solomon MA, McCoy JP Jr. CD146 (Mel-CAM), an adhesion marker of endothelial cells, is a novel marker of lymphocyte subset activation in normal peripheral blood. Blood. 2005:106:2923-4.

4. Dagur PK, Biancotto A, Stansky E, Sen HN, Nussenblatt RB, McCoy JP. Secretion of interleukin-17 by CD8+ T cells expressing CD146 (MCAM) Clin Immunol. 2014;152:36-47.

5. Dagur PK, Biancotto A, Wei L, Sen HN, Yao M, Strober W, Nussenblatt RB, McCoy JP Jr. MCAM-expressing CD4(+) T cells in peripheral blood secrete IL-17A and are significantly elevated in inflammatory autoimmune diseases. J Autoimmun. 2011;37:319-27.

6. Elshal MF, Khan SS, Raghavachari N, Takahashi Y, Barb J, Bailey JJ, Munson PJ, Solomon MA, Danner RL, McCoy JP Jr. A unique population of effector memory lymphocytes identified by CD146 having a distinct immunophenotypic and genomic profile. BMC Immunol. 2007:8:29.

7. Guezguez B, Vigneron P, Lamerant N, Kieda C, Jaffredo T, Dunon D. Dual role of melanoma cell adhesion molecule (MCAM)/CD146 in lymphocyte endothelium interaction: MCAM/CD146 promotes rolling via microvilli induction in lymphocyte and is an endothelial adhesion receptor. J Immunol. 2007;179:6673-85.
8. Dagur PK, Tatlici G, Gourley M, Samsel L, Raghavachari N, Liu P, Liu D, McCoy JP Jr. CD146+ T lymphocytes are increased in both the peripheral circulation and in the synovial effusions of patients with various musculoskeletal diseases and display pro-inflammatory gene profiles. Cytometry B Clin Cytom. 2010;78:88-95.

9. Hadjinicolaou AV, Wu L, Fang B, Watson PA, Hall FC, Busch R. Relationship of CD146 expression to activation of circulating T cells: exploratory studies in healthy donors and patients with connective tissue diseases. Clin Exp Immunol. 2013;174:73-88.

10. Kamiyama T, Watanabe $H$, lijima M, Miyazaki A, Iwamoto $\mathrm{S}$. Coexpression of CCR6 and CD146 (MCAM) is a marker of effector memory T-helper 17 cells. J Dermatol. 2012;39:838-42.

11. Mehta NNDP, Rose SM, Naik HB, Stansky E, Doveikis J, Biancotto A, Playford MP, McCoy JP. IL-17A production in human psoriatic blood and lesions by CD146+ T cells. J Invest Dermatol. 2014; in press.

12. Larochelle C, Cayrol R, Kebir H, Alvarez II, Lecuyer MA, Ifergan I, Viel E, Bourbonniere L, Beauseigle D, Terouz S, et al. Melanoma cell adhesion molecule identifies encephalitogenic $T$ lymphocytes and promotes their recruitment to the central nervous system. Brain. 2012;135:2906-24.

13. Korn T, Bettelli E, Oukka M, Kuchroo VK. IL-17 and Th17 cells. Annu Rev Immunol. 2009;27:485-517.

14. Facco M, Cabrelle A, Teramo A, Olivieri V, Gnoato M, Teolato S, Ave E, Gattazzo C, Fadini GP, Calabrese F, et al. Sarcoidosis is a Th1/Th17 multisystem disorder. Thorax. 2011;66:144-50.

15. Hu Y, Shen F, Crellin NK, Ouyang W. The IL-17 pathway as a major therapeutic target in autoimmune diseases. Ann N Y Acad Sci. 2011;1217:60-76.

16. Annunziato F, Cosmi L, Santarlasci V, Maggi L, Liotta F, Mazzinghi B, Parente E, Fili L, Ferri S, Frosali F, et al. Phenotypic and functional features of human Th17 cells. J Exp Med. 2007;204:1849-61.

17. Bettelli E, Carrier Y, Gao W, Korn T, Strom TB, Oukka M, Weiner HL, Kuchroo VK. Reciprocal developmental pathways for the generation of pathogenic effector TH17 and regulatory T cells. Nature. 2006;441:235-8.

18. Chen Z, O'Shea JJ. Th17 cells: a new fate for differentiating helper $T$ cells. Immunol Res. 2008:41:87-102.

19. Kolls JK, Linden A. Interleukin-17 family members and inflammation. Immunity. 2004:21:467-76.

20. Sallusto F, Zielinski CE, Lanzavecchia A. Human Th17 subsets. Eur J Immunol. 2012:42:2215-20

21. Sundrud MS, Trivigno C. Identity crisis of Th17 cells: many forms, many functions, many questions. Semin Immunol. 2013;25:263-72.

22. Bengsch B, Seigel B, Flecken T, Wolanski J, Blum HE, Thimme R. Human Th17 cells express high levels of enzymatically active dipeptidylpeptidase IV (CD26). J Immunol. 2012;188:5438-47.

23. Kondo T, Takata H, Matsuki F, Takiguchi M. Cutting edge: phenotypic characterization and differentiation of human CD8+ T cells producing IL-17. J Immunol. 2009;182:1794-8.

24. Maggi L, Santarlasci V, Capone M, Peired A, Frosali F, Crome SQ, Querci V, Fambrini M, Liotta F, Levings MK, et al. CD161 is a marker of all human IL17-producing T-cell subsets and is induced by RORC. Eur J Immunol. 2010; 40:2174-81.

25. Singh SP, Zhang HH, Foley JF, Hedrick MN, Farber JM. Human T cells that are able to produce IL-17 express the chemokine receptor CCR6. I Immunol. 2008:180:214-21.

26. Zuniga LA, Jain R, Haines $C$, Cua DJ. Th17 cell development: from the cradle to the grave. Immunol Rev. 2013;252:78-88.

27. Li W, Liu L, Gomez A, Zhang J, Ramadan A, Zhang Q, Choi SW, Zhang P, Greenson JK, Liu C, et al. Proteomics analysis reveals a Th17-prone cell population in presymptomatic graft-versus-host disease. JCl Insight. 2016;1.

28. Despoix N, Walzer T, Jouve N, Blot-Chabaud M, Bardin N, Paul P, Lyonnet L, Vivier E, Dignat-George F, Vely F. Mouse CD146/MCAM is a marker of natural killer cell maturation. Eur J Immunol. 2008;38:2855-64.

29. Annunziato F, Cosmi L, Liotta F, Maggi E, Romagnani S. Human Th17 cells: are they different from murine Th17 cells? Eur J Immunol. 2009;39:637-40.

30. Annunziato F, Romagnani S. Do studies in humans better depict Th17 cells? Blood. 2009:114:2213-9.

31. Geginat J, Lanzavecchia A, Sallusto F. Proliferation and differentiation potential of human CD8+ memory T-cell subsets in response to antigen or homeostatic cytokines. Blood. 2003;101:4260-6.

\section{Publisher's Note}

Springer Nature remains neutral with regard to jurisdictional claims in published maps and institutional affiliations. 